\title{
Nomogram for predicting survival in patients with pancreatic cancer
}

This article was published in the following Dove Press journal:

OncoTargets and Therapy

\section{Wei Song \\ Dong-Liu Miao \\ Lei Chen}

Department of Intervention and Vascular Surgery, Affiliated Suzhou Hospital of Nanjing Medical

University, Suzhou Municipal Hospital,

Suzhou Cancer Medical Center,

Suzhou, People's Republic of China
Correspondence: Lei Chen Department of Intervention and Vascular Surgery, Affiliated Suzhou Hospital of Nanjing Medical University, Suzhou Municipal Hospital, Suzhou Cancer Medical Center, No 16, Baita West Road, Suzhou 21500I, People's Republic of China Tel/fax +865I262364043 Email chenleia229@163.com
Background: The purpose of this study was to develop a nomogram to predict cancer-specific survival (CSS) in pancreatic cancer (PC).

Patients and methods: We used the Surveillance, Epidemiology, and End Results (SEER) database to analyze 53,028 patients diagnosed with PC from 2004 to 2014 and randomly divided them into the training $(n=26,583)$ cohort and validation $(n=26,445)$ cohort. Univariate and multivariate analyses were used to select independent prognostic factors. We used significant prognostic factors for constructing a nomogram based on Cox regression analyses. Validation of the nomogram was assessed by discrimination and calibration.

Results: According to the multivariate models of training cohort, a nomogram that combined age, race, tumor location, marital status, tumor size, TNM stage, tumor grade, and surgery was constructed for predicting CSS. The internally validated and externally validated $\mathrm{C}$-indexes were 0.741 and 0.734 , respectively. The calibration curves showed that the nomogram was able to predict 1-, 3-, and 5-year CSS accurately.

Conclusion: A nomogram effectively predicts survival in patients with PC. This prognostic model may be considered for use in clinical practice.

Keywords: pancreatic cancer, nomogram, SEER, cancer-specific survival, prognosis

\section{Introduction}

Pancreatic cancer (PC) is known as the most intractable type of cancer and the fourth leading cause of cancer-related death worldwide. ${ }^{1}$ Radical resection remains the only established curative treatment for PC, but the nonspecific symptoms and high recurrence rate after curative resection are a major problem. Moreover, it is usually diagnosed at an advanced stage; most of the patients can no longer be considered candidates for curative resection. ${ }^{2,3}$ Despite significant recent developments in surgical techniques and adjuvant therapy, the overall prognosis of $\mathrm{PC}$ remains poor. Data from the Surveillance, Epidemiology, and End Results (SEER) program (2006-2012) demonstrated that the 5-year survival of patients with $\mathrm{PC}$ is $7.7 \%$. Therefore, accurate estimates of prognosis of PC patients based on clinicopathologic factors could help clinicians implement better therapeutic strategies.

Presently, prognostic predictions and treatment strategies for PC patients are based on the American Joint Committee on Cancer (AJCC) TNM staging system., This classification system assessing PC based on the depth of invasion, number of metastasis nodes, and the status of distant metastasis has been widely used to predict the survival of patients with PC. However, survival may be different even in patients with the same AJCC stage. In fact, other patient-specific factors such as age, race, marital status, tumor size, and differentiation are associated with survival in multiple cancers. ${ }^{6,7}$ 
Therefore, a more refined staging system that combines the tumor characteristics and host status is needed.

Nomograms have been accepted as a reliable alternative tool that can help clinicians make easy individual predictions. ${ }^{8-10}$ Compared to the AJCC TNM staging system, the individual survival rate can be estimated more accurately by incorporating clinically important variables. ${ }^{11,12}$ Nevertheless, nomograms for predicting the survival of patients with PC have not yet been fully developed.

In the present study, we aimed to develop a prognostic nomogram based on large population data from the SEER cancer registry program to better predict individualized survival in patients with PC.

\section{Patients and methods}

\section{Patient population and study design}

The SEER program of the US National Cancer Institute (NCI) was used as the data source for the present population-based investigation. The SEER program captures $\sim 97 \%$ of incident cancers, and the 17 SEER tumor registries encompass $\sim 28 \%$ of the US population. ${ }^{13}$ SEER program collects information on cancer incidence, prevalence, survival, and mortality of patients with cancer.

We used the SEER program to identify patients who were diagnosed with PC from 2004 to 2014. A total of 78,665 patients were initially screened. The criteria for inclusion were listed as follows: 1) no history of malignancy; 2) the diagnosis with PC as the first and only cancer diagnosis; 3 ) active follow-up with complete date and known outcome; and 4 ) reporting clinicopathological information (age, race, sex, tumor location, tumor size, marital status, TNM stage, tumor grade, and therapy). Patients were excluded if age at diagnosis was $<18$ years, had unknown survival time, and had multiple primary cancers. The eligible patients were randomly divided into a training $(n=26,583)$ cohort and a validation $(n=26,445)$ cohort.

This study was based on public data from the SEER database; we obtained permission to access research data files with the reference number 10091-Nov 2016. This study was approved by the ethics committee of the Suzhou Municipal Hospital. The data did not include the use of human subjects or personal identifying information. Thus, no informed consent was required for this part of the study.

\section{Study variables}

There were several controlled study variables, including demographics (sex, age, and race), TNM stage, tumor location (pancreatic head, body, and tail), tumor size, tumor grade (well-differentiated, moderately differentiated, poorly differentiated, undifferentiated, not differentiated/unknown), histologic type, treatment, and marital status at the time of diagnosis. The primary end point was cancer-specific survival (CSS), which was calculated from the date of diagnosis to the date of death from PC. Deaths attributed to PC were treated as events, while deaths from other causes were treated as censored observations. Widowed, single, and separated/ divorced patients were classified as unmarried. Cancer stages were based on the sixth edition of the AJCC/TNM staging system. Meanwhile, as the sixth edition of the staging system was published in 2004, we limited our study to between 2004 and 2014.

\section{Statistical analyses \\ Construction of the nomogram}

Baseline patient demographics and disease features were compared using the Student's $t$-test or the chi-square test, as appropriate. The Kaplan-Meier method was applied to assess survivor functions. The log-rank test was used to test differences between survival curves. Cox proportional hazards multivariable regression was used to assess the independent effects of the univariate prognostic factors on CSS. A graphical nomogram, derived from the multivariate logistic regression model, was constructed using the R statistical package rms (R Foundation for Statistical Computing, Vienna, Austria).

\section{Validation of the nomogram}

Validations were conducted both internally (training cohort) and externally (validation cohort) by discrimination and calibration and using bootstrap resampling (1,000 resamples). Discrimination between survival probability and actual observations was evaluated using the $\mathrm{C}$-index. The value of the C-index fluctuated between 0.5 and 1.0 , with 0.5 representing random chance and 1.0 representing a totally corrected discrimination. ${ }^{14}$ We constructed a calibration plot to determine whether the predicted survival and actual survival were in concordance. All statistical analyses were performed by the statistical software package SPSS for Windows, version 23 (IBM Corporation, Armonk, NY, USA) and the R software version 3.13 (http://www.r-project. org/). All $P$-values were two sided, and $P$-values $<0.05$ were considered as statistically significant.

\section{Results}

\section{Patient characteristics}

The present study identified 53,028 eligible PC patients between 2004 and 2014, including 26,583 patients in the 
training cohort and 26,445 patients in the validation cohort. The flow diagram of the study selection process is shown in Figure 1. Of these patients, 27,027 (51.0\%) were male and $26,001(49.0 \%)$ were female. The most common tumor location was pancreatic head (65.2\%). The majority of patients in both cohorts were elderly patients ( $>60$ years), were married, had median tumor size $(3-5 \mathrm{~cm})$, and had IV stage tumor. In both cohorts, most patients did not receive surgery. Patient demographics and pathological characteristics are listed in Table 1.

\section{Factors associated with CSS}

Data on sex, age, race, marital status, tumor location, tumor size, tumor grade, TNM stage, and surgery were collected in the training cohort. These variables other than sex were identified as significant risk factors for poor survival in univariate analysis (Table 2). When performing multivariate analysis with Cox regression, seven factors were identified as independent prognostic factors, including age, tumor location, tumor size, marital status, TNM stage, tumor grade, and surgery. These variables were then incorporated into the nomogram.

\section{Nomogram}

Based on the reduced multivariate models of the training cohort, a nomogram that combined all the important independent factors was constructed for predicting 1-, 3-, and 5-year CSS (Figure 2). This model demonstrated that the tumor grade contributed most to prognosis, followed by the surgery, TNM stage, age, tumor size, marital status, and tumor location. Every factor was given a score on the

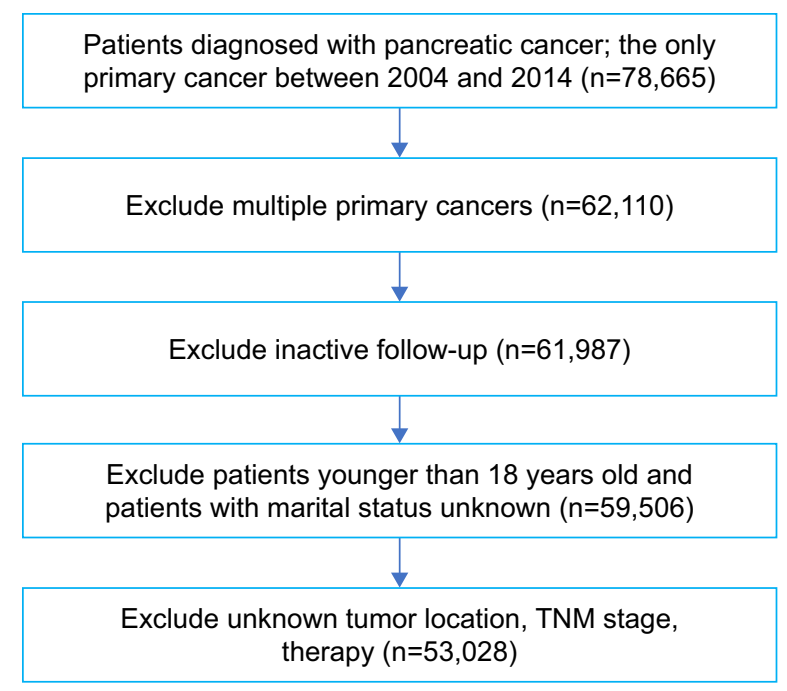

Figure I Flow diagram of the study selection process.
Table I Patient demographics and pathological characteristics

\begin{tabular}{|c|c|c|c|}
\hline \multirow[t]{2}{*}{ Variables } & \multirow{2}{*}{$\begin{array}{l}\text { All patients } \\
(\mathrm{N}=53,028) \\
\mathrm{n}(\%)\end{array}$} & \multirow{2}{*}{$\begin{array}{l}\text { Training cohort } \\
(n=26,583) \\
n(\%)\end{array}$} & \multirow{2}{*}{$\begin{array}{l}\text { Validation cohort } \\
(n=26,445) \\
n(\%)\end{array}$} \\
\hline & & & \\
\hline \multicolumn{4}{|l|}{ Sex } \\
\hline Male & $27,027(51.0)$ & |3,627 (5 . .3) & $13,400(50.7)$ \\
\hline Female & $26,001(49.0)$ & $12,956(48.7)$ & I3,045 (49.3) \\
\hline \multicolumn{4}{|l|}{ Age (years) } \\
\hline$\leq 60$ & I4,925 (28.I) & $7,537(28.4)$ & $7,388(27.9)$ \\
\hline$>60$ & 38,103 (7I.9) & I9,046 (7I.6) & $19,057(72.1)$ \\
\hline \multicolumn{4}{|l|}{ Race } \\
\hline White & $42,072(79.3)$ & $21,17 \mid(79.6)$ & $20,901(79.0)$ \\
\hline Black & $6,608(12.5)$ & $3,273(12.3)$ & $3,335(12.6)$ \\
\hline Other ${ }^{\mathrm{a}}$ & $4,348(8.2)$ & $2,139(8.1)$ & $2,209(8.4)$ \\
\hline \multicolumn{4}{|l|}{ Marital status } \\
\hline Married & $30,188(56.9)$ & I5,205 (57.2) & $14,983(56.7)$ \\
\hline Unmarried & $22,840(43.1)$ & I I,378 (42.8) & I I,462 (43.3) \\
\hline \multicolumn{4}{|c|}{ Tumor location in the pancreas } \\
\hline Head & $34,582(65.2)$ & $17,408(65.5)$ & $17, \mid 74(64.9)$ \\
\hline Body & $8,662(16.3)$ & $4,255(16.0)$ & $4,407(16.7)$ \\
\hline Tail & $9,784(18.5)$ & $4,920(18.5)$ & $4,864(18.4)$ \\
\hline \multicolumn{4}{|c|}{ Tumor size $(\mathrm{cm})$} \\
\hline$<3$ & $|2,15|(22.9)$ & $6,107(23.0)$ & $6,044(22.9)$ \\
\hline $3-5$ & $22,467(42.4)$ & I I,306 (42.5) & $|I, 16|(42.2)$ \\
\hline$>5$ & II,345 (2I.4) & $5,697(21.4)$ & $5,648(21.4)$ \\
\hline Unknown & $7,065(13.3)$ & $3,473(\mid 3.1)$ & $3,592(13.6)$ \\
\hline \multicolumn{4}{|c|}{ AJCC TNM stage } \\
\hline I & $4,431(8.4)$ & $2,282(8.6)$ & $2,149(8.1)$ \\
\hline II & $15,850(29.9)$ & 7,85I (29.5) & $7,999(30.2)$ \\
\hline III & $5,03 \mid(9.5)$ & $2,590(9.7)$ & $2,44 I(9.2)$ \\
\hline IV & $27,716(52.3)$ & $13,860(52.1)$ & $13,856(52.4)$ \\
\hline \multicolumn{4}{|l|}{ Grade } \\
\hline I & $2,759(5.2)$ & I,386 (5.2) & $\mathrm{I}, 373(5.2)$ \\
\hline ॥ & $8,227(15.5)$ & $4,08 \mathrm{I}(\mathrm{I} 5.4)$ & $4,146(15.7)$ \\
\hline III & $7,858(14.8)$ & $3,918(14.7)$ & $3,940(14.9)$ \\
\hline IV & $355(0.7)$ & $173(0.7)$ & $182(0.7)$ \\
\hline Unknown & $33,829(63.8)$ & I7,025 (64.0) & $16,804(63.5)$ \\
\hline \multicolumn{4}{|l|}{ Therapy } \\
\hline Surgery & II,338 (2I.4) & $5,645(21.2)$ & $5,693(21.5)$ \\
\hline No surgery & $41,690(78.6)$ & $20,938(78.8)$ & $20,752(78.5)$ \\
\hline
\end{tabular}

Note: ${ }^{O} O$ ther includes American Indian/Alaska native, Asian/Pacific Islander, and unknown.

Abbreviation: AJCC, American Joint Committee on Cancer.

points scale. By summing and locating the scores on the total score scale, we could predict the possibility of 1-, 3-, and 5-year survival.

\section{Nomogram validation}

The internal validation demonstrated that the nomogram can accurately predict the CSS with a C-index of 0.741 . Similarly, the C-index was 0.734 in the external validation. The calibration plots showed an excellent agreement between the predicted and observed values for the 1-, 3-, and 5-year CSS in not only the training cohort but also the validation cohort (Figures 3 and 4). 
Table 2 Univariate and multivariate analyses of CSS in the training cohort

\begin{tabular}{|c|c|c|c|}
\hline \multirow[t]{2}{*}{ Variable } & \multirow{2}{*}{$\begin{array}{l}\text { Univariate } \\
\text { analysis } \\
\text { P-value }\end{array}$} & \multicolumn{2}{|l|}{ Multivariate analysis } \\
\hline & & HR (95\% CI) & $P$-value \\
\hline Sex & 0.586 & & $\mathrm{NI}$ \\
\hline \multicolumn{4}{|l|}{ Male } \\
\hline \multicolumn{4}{|l|}{ Female } \\
\hline Age (years) & $<0.001$ & & \\
\hline$\leq 60$ & & Reference & \\
\hline$>60$ & & $1.430(1.356-1.509)$ & $<0.001$ \\
\hline Race & $<0.001$ & & \\
\hline White & & Reference & \\
\hline Black & & $1.040(0.964-1.123)$ & 0.313 \\
\hline Other ${ }^{\mathrm{a}}$ & & $0.98 \mid(0.897-1.072)$ & 0.671 \\
\hline Marital status & $<0.00 \mathrm{I}$ & & \\
\hline Married & & Reference & \\
\hline Unmarried & & 1.191 (I.I32-1.252) & $<0.001$ \\
\hline Tumor location & $<0.001$ & & \\
\hline \multicolumn{4}{|l|}{ in the pancreas } \\
\hline Head & & Reference & \\
\hline Body & & $0.834(0.772-0.900)$ & $<0.001$ \\
\hline Tail & & $0.886(0.823-0.954)$ & 0.001 \\
\hline Tumor size $(\mathrm{cm})$ & $<0.00 \mathrm{I}$ & & \\
\hline$<3$ & & Reference & \\
\hline $3-5$ & & $1.144(1.078-1.214)$ & $<0.001$ \\
\hline$>5$ & & $1.275(1.187-1.369)$ & $<0.001$ \\
\hline AJCC TNM stage & $<0.001$ & & \\
\hline I & & Reference & \\
\hline II & & 1.438 (I.306-I.583) & $<0.001$ \\
\hline III & & 1.560 (I.378-I.767) & $<0.001$ \\
\hline IV & & $2.226(2.004-2.472)$ & $<0.001$ \\
\hline Grade & $<0.001$ & & \\
\hline I & & Reference & \\
\hline II & & $1.766(1.624-1.921)$ & $<0.001$ \\
\hline III & & $2.322(2.135-2.526)$ & $<0.001$ \\
\hline IV & & $2.039(1.695-2.454)$ & $<0.001$ \\
\hline Surgery & $<0.001$ & & \\
\hline Yes & & Reference & \\
\hline No & & $2.792(2.613-2.982)$ & $<0.001$ \\
\hline
\end{tabular}

Note: ${ }^{a}$ Other includes American Indian/Alaska native, Asian/Pacific Islander, and unknown.

Abbreviations: HR, hazard ratio; CSS, cancer-specific survival; NI, not included in the multivariate survival analysis; AJCC, American Joint Committee on Cancer.

\section{Discussion}

The nomogram, as a statistical tool, can provide the most accurate predictions by a simple graphical presentation. ${ }^{15,16}$ The nomogram is simple, easy to understand, and easy to apply to clinical practice. Moreover, the model can achieve individualized predictions, and thus, clinicians can use the tool to assess patients for their participation in clinical trials. To date, several nomograms have been constructed for predicting prognosis of PC patients. ${ }^{17-19}$ Vernerey et a ${ }^{18}$ identified five parameters predicting OS before chemotherapy in 442 locally advanced PC patients. Hamada et a ${ }^{19}$ evaluated six parameters predicting survival of nonresectable PC patients. Nevertheless, all of these nomograms were based on very limited cases and variables. Therefore, a nomogram for PC with universal applicability still needs to be further validated. In fact, inclusion of sufficient data can improve the accuracy of the nomogram. Therefore, we developed the nomogram predicting the 1-, 3-, and 5-year CSS for PC based on a larger population in the SEER database.

In order to ensure the predictive accuracy of the nomogram, the Kaplan-Meier method and the Cox proportional hazards regression were used to select the factors used in developing the CSS nomogram. Moreover, C-index and calibration plots were also used to assess the predictive accuracy of the model. All nomogram C-indexes were $>0.7$, indicating excellent agreement between predicted and actual survival.

Our model is easy to use in comparison with the widely used TNM staging and has the capability to provide quantitative prognosis to individual patients. First, according to the contribution degree of each factor in the regression model, the influence score of each factor is given, and then, the total score of an individual is calculated. Consider, for example, two stage III PC patients: the first patient who is 65 -years old is diagnosed with a grade III tumor of $4 \mathrm{~cm}$ and the other patient is 55 -years old and is diagnosed with a grade II tumor of $2 \mathrm{~cm}$. Using nomograms, the two patients have 1-year CSS probabilities of $52 \%$ and $76 \%$, respectively (Table 3 ). However, according to TNM staging, ${ }^{20}$ both of the two patients are categorized with stage III tumor, which shows the same result.

We identified seven clinicopathological characteristics that can predict CSS for patients with PC, including age, tumor location, marital status, tumor size, TNM stage, tumor grade, and surgery, which are in line with previous studies. ${ }^{17-19}$ Several studies demonstrated that age is an important prognostic factor, ${ }^{21-23}$ although the exact mechanism remains unclear. Accumulated evidence showed that Black PC patients were at significantly greater risk of mortality and that Black ethnicity is associated with decreased survival. ${ }^{7,24,25}$ Our result confirmed that Black patients have the lowest CSS than other patients. Carbohydrate antigen 19-9 (CA 19-9) is widely recognized as a prognostic factor for PC. ${ }^{26-28}$ However, patients with negative red blood cell phenotypes on both Lewis A and B antigens cannot secrete CA 19-9 into their serum. In order to ensure use of the nomogram for the general population, we excluded this variable in the model but included the tumor size, which was positively associated with CA 19-9. ${ }^{19,29,30}$

Our study has several merits. Compared with previous PC nomograms, our model was constructed based on a large 


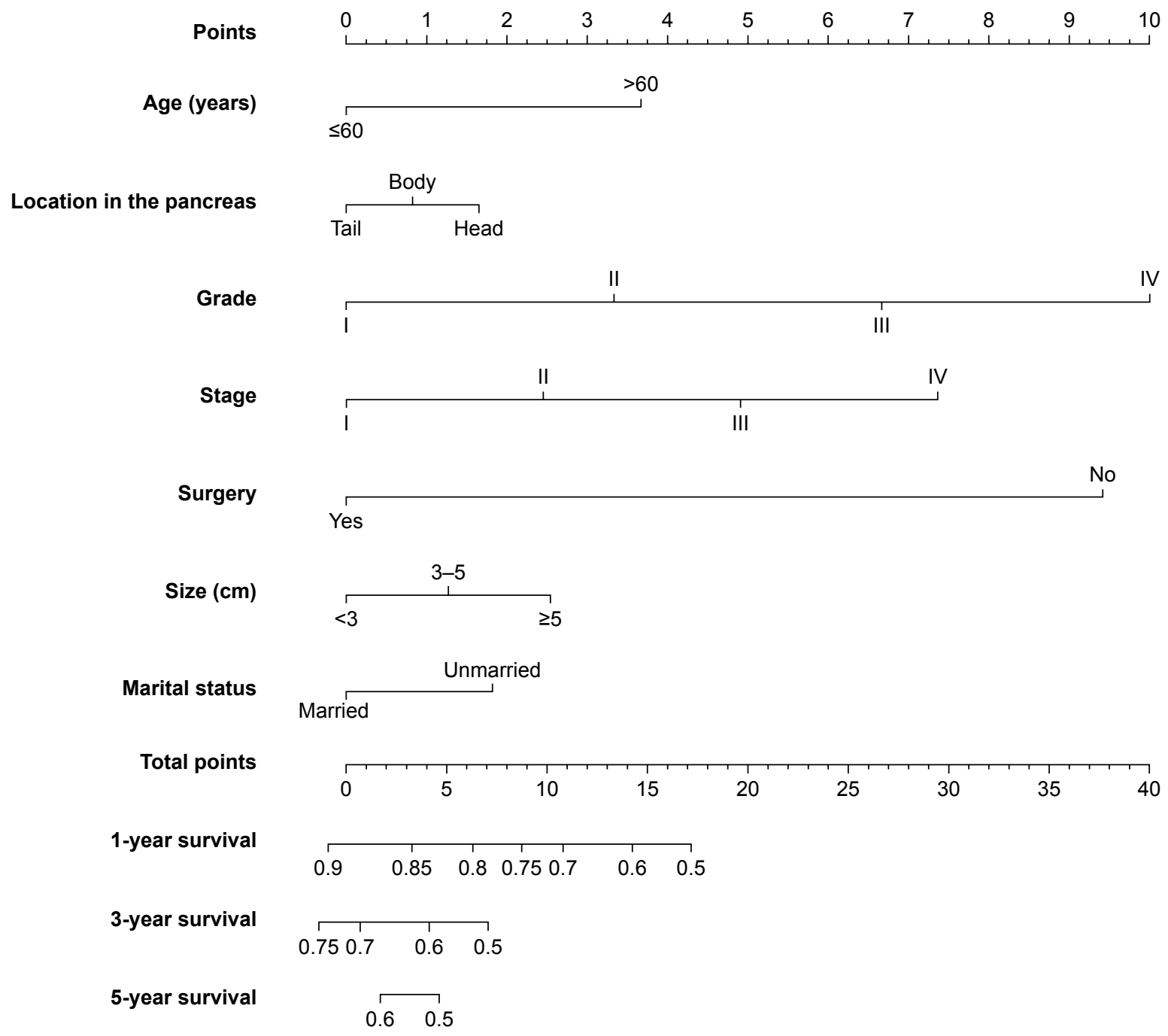

Figure 2 Nomogram for predicting 1-, 3-, and 5-year pancreatic CSS

Abbreviation: CSS, cancer-specific survival.
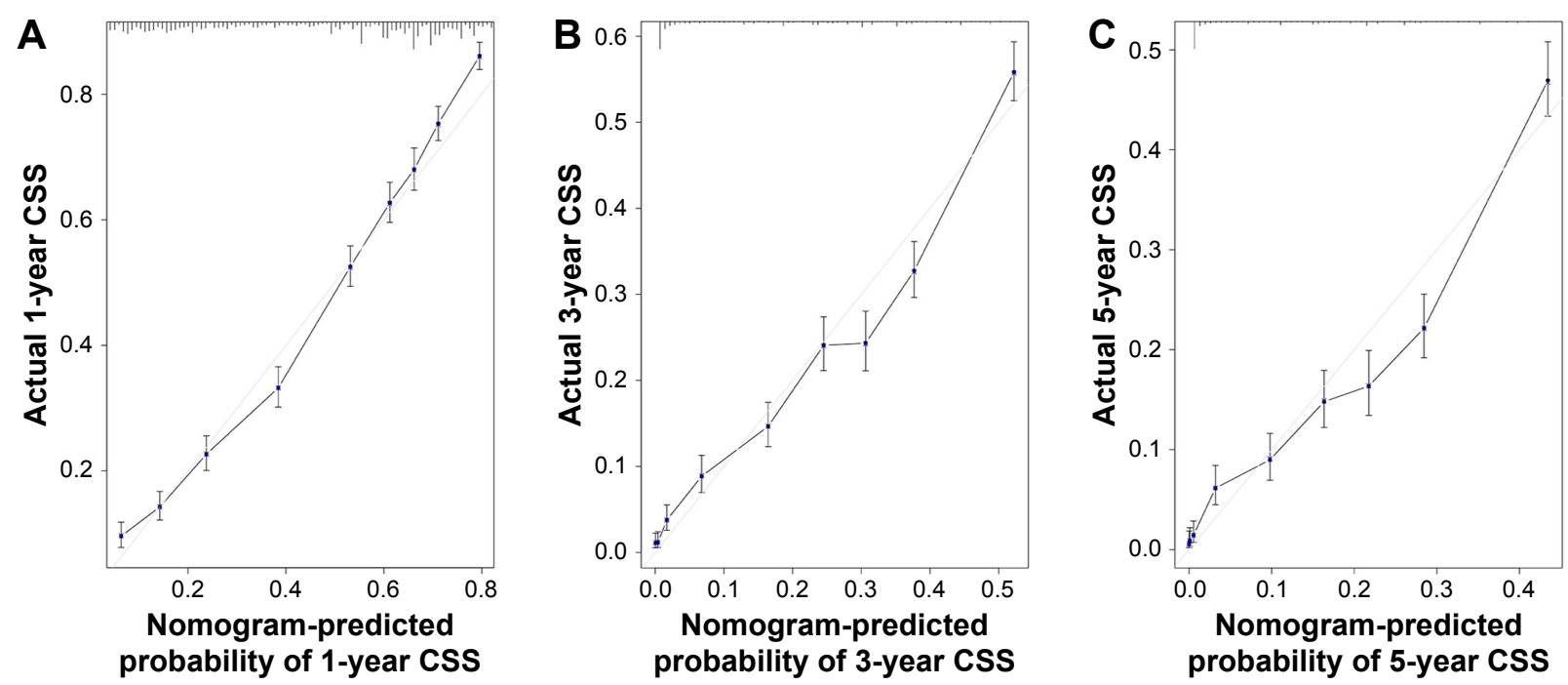

Figure 3 Internal calibration plot.

Note: (A) I-year, (B) 3-year, and (C) 5-year CSS nomogram calibration curves.

Abbreviation: CSS, cancer-specific survival. 

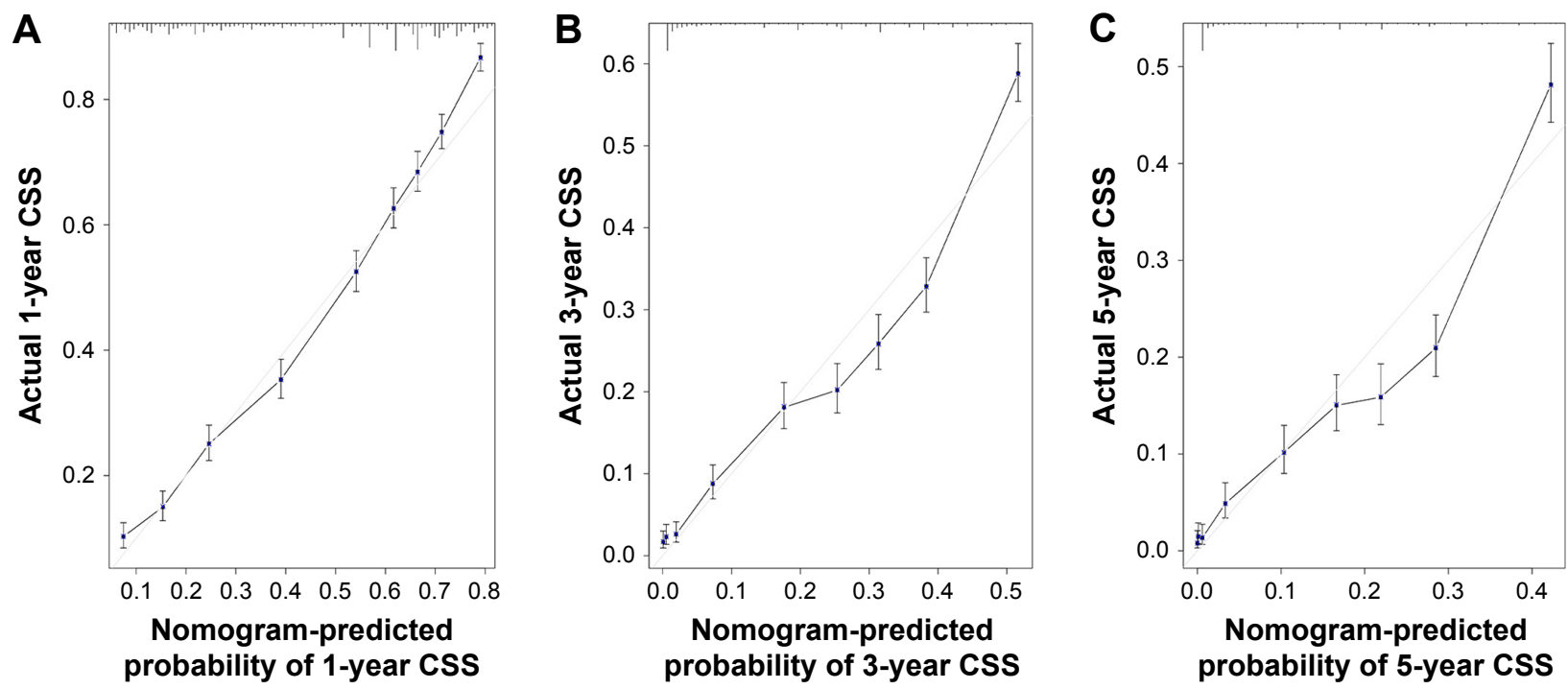

Figure 4 External calibration plot.

Note: (A) I-year, (B) 3-year, and (C) 5-year CSS nomogram calibration curves.

Abbreviation: CSS, cancer-specific survival.

population-based cohort which could improve the accuracy of the nomogram. Moreover, discrimination and calibration emphasized satisfaction in the presentation and validity of the model.

Furthermore, our nomogram identified nine variables that can be easily obtained. These variables reflect the common status of the patients and disease activity, thus providing clinically relevant information in PC. They also enhance the relevance of the tools developed.

Nevertheless, our study has several limitations. First, the SEER database did not provide data on radiotherapy and chemotherapy, which might result in bias. Moreover, data on several important clinicopathological parameters were not complete, decreasing the number of eligible cases. Second, as

Table 3 Comparison of two stage III PC patients in terms of variable and I-year CSS

\begin{tabular}{|c|c|c|c|c|c|c|}
\hline \multirow[t]{2}{*}{ Variable } & \multicolumn{3}{|c|}{ Patient A } & \multicolumn{3}{|c|}{ Patient B } \\
\hline & Value & Points & $\begin{array}{l}\text { I-year } \\
\text { CSS }\end{array}$ & Value & Points & $\begin{array}{l}\text { I-year } \\
\text { CSS }\end{array}$ \\
\hline Age (years) & 65 & 3.75 & & 55 & 0 & \\
\hline \multicolumn{7}{|l|}{ Marital status } \\
\hline \multicolumn{7}{|l|}{ Tumor location } \\
\hline Tumor size $(\mathrm{cm})$ & 4 & 1.25 & & 2 & 0 & \\
\hline AJCC TNM stage & III & 5 & & III & 5 & \\
\hline Grade & III & 6.75 & & II & 3.25 & \\
\hline \multicolumn{7}{|l|}{ Surgery } \\
\hline Total & & 16.75 & $52 \%$ & & 8.25 & $76 \%$ \\
\hline
\end{tabular}

Abbreviations: PC, pancreatic cancer; CSS, cancer-specific survival; AJCC, American Joint Committee on Cancer. this nomogram was based on the SEER database, some potential predictive variables such as pain, albumin, C-reactive protein, neutrophil-to-lymphocyte ratio, and platelet-to-lymphocyte ratio were not included. ${ }^{31-33}$ Third, TNM classification data were not available until 2004. Therefore, we were not able to predict a longer survival time.

\section{Conclusion}

We developed and validated a prognostic nomogram based on a population-based database predicting survival for patients with PC. This nomogram could help clinicians to calculate an individualized survival prediction and provide more individualized treatment.

\section{Acknowledgment}

The authors would like to thank the SEER program for providing open access to the database.

\section{Author contributions}

WS and LC conceived and designed the study. WS and D-LM searched databases and collected the data. WS and LC analyzed and interpreted the data. WS, D-LM, and LC wrote the manuscript. All authors contributed toward data analysis, drafting and critically revising the paper and agree to be accountable for all aspects of the work.

\section{Disclosure}

The authors report no conflicts of interest in this work. 


\section{References}

1. Siegel R, Naishadham D, Jemal A. Cancer statistics, 2013. CA Cancer J Clin. 2013;63(1):11-30.

2. Li D, Xie K, Wolff R, Abbruzzese JL. Pancreatic cancer. Lancet. 2004;363(9414):1049-1057.

3. Ye S. Prognostic performance of inflammation-based prognostic indices in patients with pancreatic ductal adenocarcinoma. Tumori. 2016;234(6):758-768.

4. Luo G, Javed A, Strosberg JR, et al. Modified staging classification for pancreatic neuroendocrine tumors on the basis of the American Joint Committee on Cancer and European Neuroendocrine Tumor Society Systems. J Clin Oncol. 2017;35(3):274-280.

5. Chatterjee D, Katz MH, Foo WC, et al. Prognostic significance of new AJCC tumor stage in patients with pancreatic ductal adenocarcinoma treated with neoadjuvant therapy. Am J Surg Pathol. 2017;41(8): 1097-1104.

6. Ouyang H, Ma W, Liu F, et al. Factors influencing survival of patients with pancreatic adenocarcinoma and synchronous liver metastases receiving palliative care. Pancreatology. 2017;17(5):773-781.

7. Wang XD, Qian JJ, Bai DS, Li ZN, Jiang GQ, Yao J. Marital status independently predicts pancreatic cancer survival in patients treated with surgical resection: an analysis of the SEER database. Oncotarget. 2016;7(17):24880-24887.

8. Cao J, Yuan P, Wang L, et al. Clinical nomogram for predicting survival of esophageal cancer patients after esophagectomy. Sci Rep. 2016;6:26684.

9. $\mathrm{Fu} \mathrm{J}, \mathrm{Wu} \mathrm{L}$, Jiang $\mathrm{M}$, et al. Clinical nomogram for predicting survival outcomes in early mucinous breast cancer. PLoS One. 2016; 11(10):e0164921.

10. Li Y, Ju J, Liu X, et al. Nomograms for predicting long-term overall survival and cancer-specific survival in patients with major salivary gland cancer: a population-based study. Oncotarget. 2017;8(15): 24469-24482.

11. Liu J, Geng Q, Liu Z, et al. Development and external validation of a prognostic nomogram for gastric cancer using the national cancer registry. Oncotarget. 2016;7(24):35853-35864.

12. Zhang ZY, Luo QF, Yin XW, Dai ZL, Basnet S, Ge HY. Nomograms to predict survival after colorectal cancer resection without preoperative therapy. BMC Cancer. 2016;16(1):658.

13. Cronin KA, Ries LA, Edwards BK. The surveillance, epidemiology, and end results (SEER) program of the National Cancer Institute. Cancer. 2014;120(suppl 23):3755-3757.

14. Wolbers M, Koller MT, Witteman JC, Steyerberg EW. Prognostic models with competing risks: methods and application to coronary risk prediction. Epidemiology. 2009;20(4):555-561.

15. Kattan MW, Leung DH, Brennan MF. Postoperative nomogram for 12-year sarcoma-specific death. J Clin Oncol. 2002;20(3):791-796.

16. International Bladder Cancer Nomogram Consortium, Bochner BH, Kattan MW, Vora KC. Postoperative nomogram predicting risk of recurrence after radical cystectomy for bladder cancer. J Clin Oncol. 2006;24(24):3967-3972.

17. White RR, Kattan MW, Haney JC, et al. Evaluation of preoperative therapy for pancreatic cancer using a prognostic nomogram. Ann Surg Oncol. 2006;13(11):1485-1492.

OncoTargets and Therapy

\section{Publish your work in this journal}

OncoTargets and Therapy is an international, peer-reviewed, open access journal focusing on the pathological basis of all cancers, potential targets for therapy and treatment protocols employed to improve the management of cancer patients. The journal also focuses on the impact of management programs and new therapeutic agents and protocols on

Submit your manuscript here: http://www.dovepress.com/oncotargets-and-therapy-journal
18. Vernerey D, Huguet F, Vienot A, et al. Prognostic nomogram and score to predict overall survival in locally advanced untreated pancreatic cancer (PROLAP). Br J Cancer. 2016;115(3):281-289.

19. Hamada T, Nakai Y, Yasunaga H, et al. Prognostic nomogram for nonresectable pancreatic cancer treated with gemcitabine-based chemotherapy. Br J Cancer. 2014;110(8):1943-1949.

20. Edge SB, Compton CC. The American Joint Committee on Cancer: the 7th edition of the AJCC cancer staging manual and the future of TNM. Ann Surg Oncol. 2010;17(6):1471-1474.

21. Shen W, Sakamoto N, Yang L. Cancer-specific mortality and competing mortality in patients with head and neck squamous cell carcinoma: a competing risk analysis. Ann Surg Oncol. 2015;22(1):264-271.

22. Skillington SA, Kallogjeri D, Lewis JS Jr, Piccirillo JF. Prognostic importance of comorbidity and the association between comorbidity and p16 in oropharyngeal squamous cell carcinoma. JAMA Otolaryngol Head Neck Surg. 2016;142(6):568-575.

23. Wray CJ, Phatak UR, Robinson EK, et al. The effect of age on racerelated breast cancer survival disparities. Ann Surg Oncol. 2013;20(8): 2541-2547.

24. Sun H, Ma H, Hong G, Sun H, Wang J. Survival improvement in patients with pancreatic cancer by decade: a period analysis of the SEER database, 1981-2010. Sci Rep. 2014;4:6747.

25. Baine M, Sahak F, Lin C, Chakraborty S, Lyden E, Batra SK. Marital status and survival in pancreatic cancer patients: a SEER based analysis. PLoS One. 2011;6(6):e21052.

26. Yang GY, Malik NK, Chandrasekhar R, et al. Change in CA 19-9 levels after chemoradiotherapy predicts survival in patients with locally advanced unresectable pancreatic cancer. J Gastrointest Oncol. 2013; 4(4):361-369.

27. Combs SE, Habermehl D, Kessel KA, et al. Prognostic impact of CA 19-9 on outcome after neoadjuvant chemoradiation in patients with locally advanced pancreatic cancer. Ann Surg Oncol. 2014;21(8):2801-2807.

28. Ballehaninna UK, Chamberlain RS. Serum CA 19-9 as a biomarker for pancreatic cancer - a comprehensive review. Indian J Surg Oncol. 2011;2(2):88-100.

29. Sakahara H, Endo K, Nakajima K, et al. Serum CA 19-9 concentrations and computed tomography findings in patients with pancreatic carcinoma. Cancer. 1986;57(7):1324-1326.

30. Tian F, Appert HE, Myles J, Howard JM. Prognostic value of serum CA 19-9 levels in pancreatic adenocarcinoma. Ann Surg. 1992;215(4): 350-355.

31. Asari S, Matsumoto I, Toyama H, et al. Preoperative independent prognostic factors in patients with borderline resectable pancreatic ductal adenocarcinoma following curative resection: the neutrophil-lymphocyte and platelet-lymphocyte ratios. Surg Today. 2016;46(5):583-592.

32. Shirai Y, Shiba H, Sakamoto T, et al. Preoperative platelet to lymphocyte ratio predicts outcome of patients with pancreatic ductal adenocarcinoma after pancreatic resection. Surgery (United States). 2015;158(2): 360-365.

33. Liu Z, Jin K, Guo M, et al. Prognostic value of the CRP/Alb ratio, a novel inflammation-based score in pancreatic cancer. Ann Surg Oncol. 2017;24(2):561-568.

patient perspectives such as quality of life, adherence and satisfaction. The manuscript management system is completely online and includes a very quick and fair peer-review system, which is all easy to use. Visit http://www.dovepress.com/testimonials.php to read real quotes from published authors.

\section{Dovepress}

